\title{
Tecnologias de informação e comunicação na educação da Odontologia: estudo transversal de uma população do sul do Brasil
}

\author{
Bruna Genari*, Jules Renan Dutra Bemfica**, Nicole Flach***, Daniel Candido Haddad****, \\ Susana Maria Werner Samuel*****, Claídes Abegg******
}

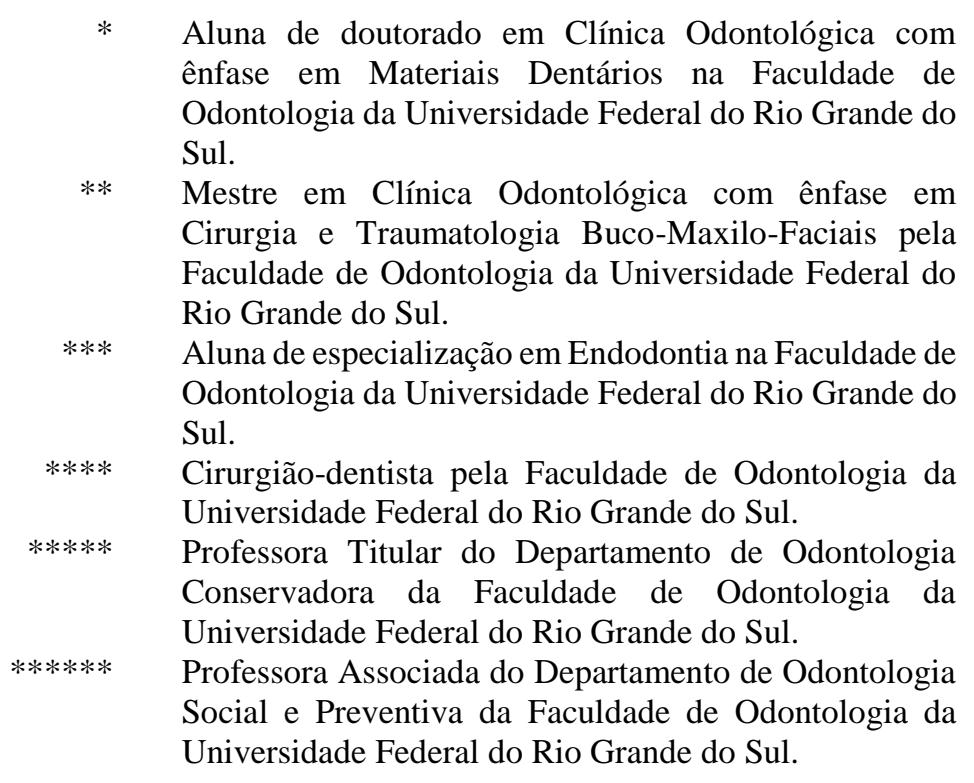

\section{RESUMO}

O objetivo do presente estudo foi avaliar as competências em relação às Tecnologias de Informação e Comunicação (TICs) de estudantes de Odontologia. Um estudo transversal foi realizado com os estudantes de Odontologia da Universidade Federal do Rio Grande do Sul, Brasil. Os dados foram coletados através de um questionário previamente validado pelo Centro de Ciências de Saúde Oral da Universidade de Malmö e submetido ao processo de adaptação transcultural para o português. Os resultados foram transformados em escores conforme literatura prévia e categorizados para as comparações.

Análise descritiva, qui-quadrado e teste exato de Fischer foram utilizados. O escore de competência em relação às TICs foi considerado muito bom e apresentou relação com o nível de escolaridade materno. Já o escore de importância das TICs foi mediano e relacionado à idade. Concluiu-se que os estudantes de Odontologia apresentaram bom domínio das ferramentas de TICs e nível moderado em relação à importância atribuída ao uso odontológico dessas tecnologias.

Descritores: Educação Baseada em Competências. Informática Aplicada à Odontologia. Educação em Odontologia. 


\section{INTRODUÇÃO}

As Tecnologias de Informação e Comunicação (TICs) têm sido utilizadas em proporções crescentes na Odontologia nos últimos anos como ferramenta para gestão clínica, uso de imagem digital, base de dados, comunicação eletrônica, educação continuada, informação à distância e como ferramenta didáticopedagógica ${ }^{1}$. Dessa forma, o domínio do uso dessas ferramentas tem se tornado necessário tanto para estudantes quanto para profissionais de Odontologia $^{2}$. Portanto, são necessárias pesquisas referentes às competências dos estudantes bem como o planejamento de intervenções a partir dos resultados destas investigações ${ }^{2,3}$.

Estudos internacionais que investigam o conhecimento e uso das TICs por estudantes de Odontologia têm revelado que a maioria deles apresenta domínio entre regular e bom dessas tecnologia ${ }^{4,5}$. Investigações sobre este tema são escassas na população estudantil brasileira, apesar do uso crescente dessas ferramentas nas universidades nos últimos anos. Por exemplo, aulas vinculadas às plataformas de Ensino à Distância como MOODLE e NAVI vêm sendo cada vez mais estimuladas nas Instituições de Ensino Superior no Brasil. Além disso, o Ministério da Saúde tem utilizado as TICs para melhorar o serviço público ${ }^{6,7}$. Um exemplo é o programa TeleSaúde, que utiliza as ferramentas tecnológicas como forma de comunicação entre profissionais da saúde de diferentes locais e professores universitários, a fim de discutir problemas de saúde, diagnósticos e tratamentos, minimizando distâncias, otimizando tempo e reduzindo custos no que se refere à educação continuada dos profissionais e melhor atendimento da população ${ }^{7}$.

O objetivo do presente estudo foi avaliar as competências em relação às TICs de estudantes de uma Faculdade de Odontologia do Sul do Brasil.

\section{MATERIAL E MÉTODOS}

Um estudo transversal foi realizado com os estudantes de Odontologia da Universidade Federal do Rio Grande do Sul, Brasil. Os dados foram coletados através de um questionário sobre TICs, para estudantes de Odontologia, validado pelo Centro de Ciências de Saúde Oral da Universidade de Malmö (Suécia). Este instrumento foi submetido ao processo de adaptação transcultural para o Português, conforme o protocolo descrito na literatura ${ }^{8}$ antes de ser utilizado.

As questões estavam organizadas em cinco grupos representando as maiores áreas de aplicação das tecnologias na educação: habilidades gerais e gerenciamento de arquivos (10 questões), uso de programas (10 questões), comunicação (8 questões), uso da internet e ferramentas de busca (5 questões) e codificação HTML (10 questões). As perguntas estavam ordenadas por nível crescente de complexidade e as respostas eram "sim" ou "não", dependendo da habilidade ou não do estudante de realizar a tarefa em questão. Duas perguntas sobre internet e ferramentas de busca apresentavam formato diferente, com cinco e sete opções de resposta, respectivamente, além de quatro perguntas sobre a importância das TICs com escala de 1 (um) a $10(\mathrm{dez})^{5}$. Além das perguntas sobre as tecnologias, havia ainda questões relacionadas à condição sóciodemográfica dos respondentes. 
O questionário autoaplicado foi administrado durante as aulas para os estudantes de Odontologia de todos os semestres presentes em sala de aula. Um total de 335 alunos fez parte do estudo, o que representou $85 \%$ dos estudantes de Odontologia da Universidade Federal do Rio Grande do Sul no ano de 2009.

Os dados coletados foram transformados em escores com base em estudo anterior ${ }^{5}$. O escore de competência em relação às TICs foi obtido com a soma das respostas positivas, podendo variar de 0 a 47. O escore da importância atribuída ao uso das TICs na vida profissional, avaliado por meio da escala de 0 a 10 foi obtido pela soma das respostas, podendo variar de 0 a 40. De acordo com estudo anterior ${ }^{5}$, escore de competência abaixo de 20 é considerado ruim, de 20 a 22 é regular/desejável e acima de 22, muito bom. Para fins de análise estatística, o escore de competência foi categorizado conforme essa classificação ${ }^{5}$ (abaixo de 20 , de 20 a 22 e acima de 22) e o escore de importância pela mediana (até 25 e acima de 25). Já os dados sociodemográficos foram dicotomizados: sexo (masculino e feminino), idade pela média (até 21 anos e acima de 21), semestre pela mediana (até $5^{\circ}$ semestre e acima do $5^{\circ}$ ), nível educacional paterna e materna (até ensino médio completo e ensino superior) e renda familiar (até 5 salários mínimos e acima de $5)$.

Os dados foram analisados por intermédio da análise descritiva. Para testar a associação entre as variáveis sóciodemográficas e o escore de competência e de importância foram utilizados os testes Qui-Quadrado e exato de Fischer, respectivamente. Essa análise foi realizada através do SPSS 16.0 (Statistical Package for the Social Sciences, Adobe Systems Inc., São José, EUA) e o nível de significância adotado foi de $5 \%$.

A participação no estudo foi voluntária e todos os participantes permaneceram no anonimato. O projeto do presente estudo foi aprovado pelo Comitê de Ética e Pesquisa da Universidade Federal do Rio Grande do Sul. Todos os participantes assinaram o Termo de Consentimento Livre e Informado.

\section{RESULTADOS}

A maioria dos participantes do estudo era do sexo feminino $(71,6 \%)$ com média de 21,9 $( \pm 2,59)$ anos de idade. Cerca de metade dos pais e mães dos estudantes não possuem ensino superior, 51,2\% e $56,3 \%$ respectivamente. Em relação à renda, mais de dois terços das famílias dos participantes possuem renda superior a cinco salários mínimos $(87,6 \%)$. Apenas $13,4 \%$ dos estudantes responderam ter tido algum tipo de curso à distância até o dia da aplicação do questionário (Tabela 1).

A média do escore de competência foi $27,2( \pm 5,9)$ (Tabela 3). Apenas $8,4 \%$ dos participantes apresentaram escore abaixo de 20, enquanto $9,6 \%$, escores entre 20 e 22 e 82,1\%, acima de 22 (Tabela 2). Esse escore mostrou-se associado com o nível educacional das mães $(\mathrm{p}=0,032)$ (Tabela 4).

A média do escore de importância atribuída às TICs foi 25,6 $( \pm 5,7)$, moda, 22 e mediana, 25 (Tabela 3). A metade dos participantes do estudo (51\%) apresentaram escore acima de 25 (Tabela 2). Esse escore mostrou-se relacionado com a idade dos alunos $(\mathrm{p}=0,04)$; os mais velhos atribuíram maior importância (Tabela 5). 
Tabela 1: Análise descritiva dos dados sociodemográficos.

\begin{tabular}{lrr}
\hline Variável & & $\mathrm{n}(\%)$ \\
\hline Sexo & Feminino & \\
& Masculino & $232(71,6)$ \\
Idade & & $92(28,4)$ \\
& Até 21 anos & \\
& Acima de 21 anos & $149(46,3)$ \\
Nível educacional paterno & $173(53,7)$ \\
Ensino fundamental incompleto & \\
Ensino fundamental & $11(3,8)$ \\
Ensino médio & $23(7,9)$ \\
Ensino superior & $115(39,5)$ \\
Nível educacional materno & $142(48,8)$ \\
Ensino fundamental incompleto & \\
Ensino fundamental & $9(3,1)$ \\
Ensino médio & $18(6,2)$ \\
Ensino superior & $101(34,5)$ \\
Renda familiar & $165(56,3)$ \\
Até 5 salários mínimos & \\
De 6 a 10 salários mínimos & $35(12,4)$ \\
De 11 a 20 salários mínimos & $101(35,8)$ \\
Acima de 20 salários mínimos & $101(35,8)$ \\
& $45(16)$ \\
\hline Total & $335(100)$ \\
\hline
\end{tabular}

Tabela 2: Análise descritiva dos escores.

\begin{tabular}{|c|c|}
\hline Escore & $\mathrm{n}(\%)$ \\
\hline \multicolumn{2}{|l|}{ Escore de competência } \\
\hline Abaixo de 20 & $28(8,4)$ \\
\hline De 20 a 22 & $32(9,6)$ \\
\hline Acima de 22 & $275(82,1)$ \\
\hline \multicolumn{2}{|c|}{ Escore de importância do uso das TIC } \\
\hline Até 25 & $171(51)$ \\
\hline Acima de 25 & $164(49)$ \\
\hline Total & $335(100)$ \\
\hline
\end{tabular}

Tabela 3: Medidas de tendência central.

\begin{tabular}{lccccc}
\hline & $\begin{array}{c}\text { Média } \\
\text { (desvio } \\
\text { padrão) }\end{array}$ & Mediana & Moda & Mínimo & Máximo \\
\hline $\begin{array}{l}\text { Idade } \\
\begin{array}{l}\text { Escore de } \\
\text { competência }\end{array}\end{array}$ & $21,9(2,6)$ & 22 & 22 & 17 & 35 \\
$\begin{array}{l}\text { Escore de } \\
\text { importância do } \\
\text { uso das TIC }\end{array}$ & $25,5(5,9)$ & 27 & 29 & 8 & 45 \\
\hline
\end{tabular}


Tabela 4: Relação entre escore de competência e dados demográficos.

\begin{tabular}{|c|c|c|c|c|c|}
\hline & \multicolumn{3}{|c|}{ Escores sociodemográficos } & \multirow{2}{*}{$\begin{array}{l}\text { Total } \\
\mathrm{n}(\%)\end{array}$} & \multirow[t]{2}{*}{$p$} \\
\hline & $\begin{array}{l}<20 \\
\mathrm{n}(\%)\end{array}$ & $\begin{array}{c}\text { De } 20 \text { a } 22 \\
\text { n }(\%)\end{array}$ & $\begin{array}{c}>22 \\
\mathrm{n}(\%)\end{array}$ & & \\
\hline Sexo & & & & & 0,894 \\
\hline Feminino & $20(8,6)$ & $23(9,9)$ & $189(81,5)$ & $232(71,6)$ & \\
\hline Masculino & $7(7,6)$ & $8(8,7)$ & $77(83,7)$ & $92(28,4)$ & \\
\hline Idade & & & & & 0,463 \\
\hline Até 21 anos & $13(8,7)$ & $17(11,4)$ & $119(79,9)$ & $149(46,3)$ & \\
\hline Acima de 21 anos & $14(8,1)$ & $13(7,5)$ & $146(84,4)$ & $173(53,7)$ & \\
\hline Semestre & & & & & 0,611 \\
\hline Até $5^{\circ}$ semestre & $19(9,6)$ & $19(9,6)$ & $160(80,8)$ & $198(59,1)$ & \\
\hline Acima de $5^{\circ}$ semestre & $9(6,6)$ & $13(9,5)$ & $115(83,9)$ & $137(40,9)$ & \\
\hline Nível educacional paterno & & & & & 0,855 \\
\hline Até ensino médio completo & $11(7,4)$ & $15(10,1)$ & $123(82,6)$ & $149(51,2)$ & \\
\hline Ensino superior completo & $12(8,5)$ & $12(8,5)$ & $118(83,1)$ & $142(48,8)$ & \\
\hline Nível educacional materno & & & & & $0,032 *$ \\
\hline Até ensino médio completo & $16(12,5)$ & $12(9,4)$ & $100(78,1)$ & $128(43,7)$ & \\
\hline Ensino superior completo & $7(4,2)$ & $15(9,1)$ & $143(86,7)$ & $165(56,3)$ & \\
\hline Renda familiar & & & & & 0,235 \\
\hline Até 5 salários mínimos & $5(14,3)$ & $2(5,7)$ & $28(8,0)$ & $35(12,4)$ & \\
\hline$>$ que 5 salários mínimos & $17(6,9)$ & $26(10,5)$ & $204(82,6)$ & $247(87,6)$ & \\
\hline
\end{tabular}

Teste qui-quadrado. * Diferença estatisticamente significante $(\mathrm{p}<0,05)$.

Tabela 5: Relação entre escore de importância atribuída às TIC e dados demográficos.

\begin{tabular}{|c|c|c|c|c|}
\hline & \multicolumn{2}{|c|}{ Escore de importância das TIC } & \multirow{2}{*}{$\begin{array}{l}\text { Total } \\
\mathrm{n}(\%)\end{array}$} & \multirow[t]{2}{*}{$\mathrm{P}$} \\
\hline & $\begin{array}{c}\text { Até } 25 \\
\mathrm{n}(\%)\end{array}$ & $\begin{array}{c}\text { Acima de } 25 \\
\mathrm{n}(\%)\end{array}$ & & \\
\hline Sexo & & & & 0,902 \\
\hline Feminino & $118(50,9)$ & $114(49,1)$ & $232(71,6)$ & \\
\hline Masculino & $48(52,2)$ & $44(47,8)$ & $92(28,4)$ & \\
\hline Idade & & & & $0,045 *$ \\
\hline Até 21 anos & $85(57,0)$ & $64(43,0)$ & $149(46,3)$ & \\
\hline Acima de 21 anos & $79(45,7)$ & $94(54,3)$ & $173(53,7)$ & \\
\hline Semestre & & & & 0,148 \\
\hline Até $5^{\circ}$ semestre & $108(54,5)$ & $90(45,5)$ & $198(59,1)$ & \\
\hline Acima de $5^{\circ}$ semestre & $63(46,0)$ & $74(54,0)$ & $137(40,9)$ & \\
\hline Nível educacional paterno & & & & 1,000 \\
\hline Até ensino médio completo & $78(52,8)$ & $71(47,7)$ & $149(51,2)$ & \\
\hline Ensino superior completo & $75(52,3)$ & $67(47,2)$ & $142(48,8)$ & \\
\hline Nível educacional materno & & & & 0,556 \\
\hline Até ensino médio completo & $70(54,7)$ & $58(45,3)$ & $128(43,7)$ & \\
\hline Ensino superior completo & $84(50,9)$ & $81(49,1)$ & $165(56,3)$ & \\
\hline Renda familiar & & & & 0,719 \\
\hline Até 5 salários mínimos & $19(54,3)$ & $16(45,7)$ & $35(12,4)$ & \\
\hline Acima de 5 salários mínimos & $124(50,2)$ & $123(49,8)$ & $247(87,6)$ & \\
\hline
\end{tabular}

Teste exato de Fischer. * Diferença estatisticamente significante $(\mathrm{p}<0,05)$. 


\section{DISCUSSÃO}

As tecnologias de informação e comunicação são um recurso valioso na educação e saúde. O presente estudo foi pioneiro na avaliação das competências de estudantes de Odontologia brasileiros em relação às TICs com questionário validado e adequado para o português. Uma competência satisfatória foi demonstrada pela maioria dos participantes do estudo, representando um recurso didático importante para ser utilizado pelos professores do ensino universitário ${ }^{5}$.

O escore de competência obtido no presente estudo está acima da faixa considerada desejável pelos autores do questionário original ${ }^{5}$. Além disso, o valor médio $(27,2 \pm 5,9)$ foi maior que o valor encontrado para estudantes gregos $(22,6 \pm 8,4)$ e suecos do quinto ano do curso de Odontologia $(18,1 \pm 8,5)^{2,5}$. A similaridade do escore de competência encontrada entre alunos brasileiros e suecos chama a atenção. A Suécia está entre os países mais avançados na questão da implementação de TICs na educação e em outros aspectos da vida cotidiana em comparação com outros países europeus ${ }^{4}$. Estudos prévios, assim como o presente estudo, indicam certa diversidade em relação a essas competências, o que poderia ser nivelado com atividades curriculares no ambiente universitário $^{4,5,9-11}$. Argumenta-se que os professores geralmente assumem que os alunos dominam as ferramentas tecnológicas por serem jovens. Entretanto, alguns estudantes usam as tecnologias apenas para atividades cotidianas de comunicação, jogos e programas de texto e não necessariamente como ferramenta de maior complexidade para uso na educação ${ }^{5}$.

A importância atribuída ao uso das TICs no presente estudo teve valor considerado mediano $(25,6 \pm 5,7)$. Já estudo prévio em país desenvolvido apresentou mais claramente opiniões positivas em relação à necessidade e importância do uso dessas tecnologias no ambiente clínico e acadêmico ${ }^{5}$. O resultado do presente estudo sugere a necessidade de demonstrar, durante o curso de Odontologia no Sul do Brasil, a importância e o impacto que as TICs podem ter no contexto clinico e acadêmico. É possível também que se tenha ciência da necessidade da implantação de recursos de tecnologias de informação e comunicação, ainda que haja alguns desafios a serem superados ${ }^{12}$.

Não se encontrou na literatura estudos avaliando a relação das competências em relação às TICs e dados sociodemográficos. No presente estudo, observou-se associação entre o escore de competência das TICs e o nível educacional materno, sugerindo maior influência da mãe na educação dos filhos. Estudos prévios têm demonstrado que o nível educacional materno está associado com outros aspectos da vida dos filhos: saúde, obesidade infantil, uso de álcool e cigarro e desempenho escolar ${ }^{13-16}$. Já a importância atribuída às TICs apresentou relação com a idade no presente estudo. Estudantes mais velhos geraram valores mais altos desse escore, sugerindo que as experiências de vida e maturidade confirmam a necessidade de uso dessas tecnologias.

A partir da literatura 
especializada, reconhece-se que questionários dependentes de autoavaliação com escala ordinal apresentam resultados difíceis de serem interpretados e vieses no sentido de superestimar as próprias competências $^{3,17}$. O questionário utilizado no presente estudo foi baseado em estudo prévio com questões diretas e respostas dicotômicas na maioria das perguntas sobre as competências, a fim de evitar vieses. Dessa forma, é possível a obtenção de uma descrição objetiva das competências estudadas 5 . Além disso, realizou-se a adaptação transcultural do instrumento para o Português conforme descrito na literatura.

Alunos que não estavam em aula não participaram do presente estudo, $o$ que poderia causar uma exclusão de um perfil específico de estudantes, que poderia influenciar o escore final. Entretanto, apenas $15 \%$ dos alunos matriculados não participaram do estudo por não estarem em aula no momento da coleta de dados. Ao mesmo tempo, os resultados encontrados não podem ser extrapolados para estudantes de outros cursos de Odontologia no Brasil em função das diferenças regionais. Assim, sugere-se a realização de mais estudos em diferentes regiões do país a fim de possibilitar comparações e se obter dados representantes dos cursos de Odontologia brasileiros.

\section{CONCLUSÕES}

Considerando as limitações do presente estudo, é possível concluir que os estudantes de uma Faculdade de Odontologia do sul do Brasil apresentaram um nível muito bom de competência em relação às TICs e nível moderado em relação à importância atribuída ao uso odontológico dessas tecnologias.

\section{REFERÊNCIAS}

1. Silveira LGGD, Doki R, Lopes PRDL, Sigulem D, Pisa IT, Schor P. Evaluation of the use of information technology by the dentist and dental academic. RGO. 2006;54(2):119-22.

2. Divaris K, Polychronopoulou A, Mattheos N. An investigation of computer literacy and attitudes amongst Greek post-graduate dental students. Eur J Dent Educ. 2007;11(3):144-47.

3. Mattheos N, Nattestad A, Schittek M, Attstrom R. A virtual classroom in undergraduate periodontology: a pilot study. Eur J Dent Educ. 2001;5(4):139-47.

4. Mattheos N, Nattestad A, Schittek M, Attström R. Computer literacy and attitudes among students in 16 European dental schools: current aspects, regional differences and future trends. Eur J Dent Educ. 2002;6(1):30-5.

5. Mattheos N, Schittek MJ, Nattestad A, Shanley D, Attström R. A comparative evaluation of computer literacy amongst dental educators and students. Eur J Dent Educ. 2005;9(1):32-6.

6. Camargo LB, Aldrigui JM, Imparato JC, Mendes FM, Wen CL, Bönecker $\mathrm{M}$ et al. E-learning used in a training course on atraumatic restorative treatment (ART) for Brazilian dentists. J Dent Educ. 2011;75(10):1396-401. 
7. Silva AS, Rizzante FA, Picolini MM, Campos KD, Corrêa CC, Franco EC et al. Bauru School of Dentistry TeleHealth League: an educational strategy applied to research, teaching and extension among applications in tele-health. J Appl Oral Sci. 2011;19(6):599-603.

8. Reichenheim ME, Moraes CL. Crosscultural adaptation of epidemiological measurement tools: a proposal for operationalization. Rev Saúde Públ. 2007;41(4):665-73.

9. Jones RB, Navin LM, Barrie J, Hillan E, Kinane D. Computer literacy among medical, nursing, dental and veterinary undergraduates. Med Educ. 1991;25(3):191-5.

10. Desjardins KS, Cook SS, Jenkins M, Bakken S. Effect of an informatics for Evidence-based Pratice Curriculum on nursing informatics competences. Int J Med Inf. 2005;74(11-12):101220.

11. Robabi H, Arbabisarjou A. Computer Literacy Among Students of Zahedan University of Medical Sciences. Glob J Health Sci. 2015;7(4):40274.

12. Jathanna VR, Jathanna RV, Jathanna $\mathrm{R}$. The awareness and attitudes of students of one indian dental school toward information technology and its use to improve patient care. Educ Health (Abingdon). 2014;27(3):2936.

13. Desai S, Alva S. Maternal education and child health: is there a strong causal relationship? Demography. 1998;35(1):71-81.

14. Baughcum AE, Chamberlin LA, Deeks CM, Powers SW, Whitaker RC. Maternal Perceptions of
Overweight Preschool Children. Pediatrics. 2000;106(6):1380-6.

15. Singhammer J, Mittelmark MB. Associations between mother's level of education and offspring's smoking and alcohol use in adulthood: a 28year longitudinal follow-up study. J Public Health. 2006;14(6):132-8.

16. Magnuson K. Maternal education and children's academic achievement during middle childhood. Dev Psychol. 2007;43(6):1497-1512.

17. Grigg PA, Stephens CD. A survey of the IT skills and attitudes of final year dental students at Bristol University in 1996 and 1997. Eur J Dent Educ. 1999;3(2):64-73.

\section{ABSTRACT \\ Information and Communication Technologies in dental education: cross-sectional study of a population of southern Brazil}

The purpose of the present study was to evaluate the competence of the students to use technologies in dental educational. A cross-sectional study was done with dental students of a university of the south of Brazil. It was used a questionnaire previously validated by the Center Oral Health Sciences of University of Malmö (Sweden) and adapted to Portuguese. The collected data were transformed into scores based on Mattheos et al. (2005). For analysis purposes, scores and sociodemographic variables were categorized. The descriptive analyses was realized, Chi Square and Exact Fischer's tests were used to test the association between the sociodemographic data and the scores regarding the Information and Communication Technologies (ICT). The mean competence score was considered very good and associated 
with education level of the mothers. The mean importance score was regular and associated with age. It was concluded that the students of a university of south of Brazil had a very good level regarding the competence of the use of ICT even though moderate level in relation to the importance was given to dental use of these technologies.

Descriptors: $\quad$ Competency-Based Education. Dental Informatics. Education, Dental.
Correspondência para:

Claídes Abegg

e-mail: claides.abegg@gmail.com

Universidade Federal do Rio Grande do

Sul, Faculdade de Odontologia,

Departamento de Odontologia

Preventiva e Social.

Rua Ramiro Barcelos 2492 - Santana

90035-003 Porto Alegre, RS 